\title{
Multiculturalidade, interculturalidade, direitos humanos e violência de género: breves notas para pensar o caso da mutilação genital feminina em Portugal e a sua abordagem*
}

\author{
Nuno Medeiros** \\ Teresa Denis***
}

\section{Resumo}

A situação de multiculturalidade é hoje uma realidade crescentemente vivida nas sociedades de acolhimento de imigrantes, onde emergem cidades que se constituem como lugares de passagem, de encontro ou mestiçagem cultural, mas também de contraste ou antagonismo social. A interculturalidade aparece como utensilio de favorecimento de vinculação à ideia de outro, promovendo, por um lado, a integração e a defesa do direito à diferença, e, por outro, evitando - ou mesmo denunciando - as estruturas de hegemonização sobre minorias. Mas esta diversidade cultural só pode ser protegida, promovida e aceite desde que não coloque em causa os direitos humanos nem provoque exclusões ou desigualdades. É neste campo, difícil e persistentemente aberto ao debate (e não raro promotor de polarizações), que nos propomos a apresentar um conjunto breve de apontamentos que contribua para pensar a mutilação genital feminina na sua indissolúvel condição de violência sobre a mulher, partindo do exemplo da realidade portuguesa actual.

Palavras-chave: Mutilação Genital Feminina, Interculturalidade, Violência de Género, Direitos Humanos, Portugal.

\footnotetext{
Recebido em 10 de outubro de 2015, aceito em 26 de fevereiro de 2019.

** Pesquisador no IHC - NOVA FCSH, Instituto de História Contemporânea da Universidade Nova de Lisboa, e no H\&TRC - Centro de Investigação em Saúde e Tecnologia; Professor do Departamento das Ciências Sociais e Humanas da Escola Superior de Tecnologia da Saúde de Lisboa do Instituto Politécnico de Lisboa, Lisboa, Portugal.nuno.medeiros@fcsh.unl.pt / http://orcid.org/0000-0001-5350-4294

${ }^{* * *}$ Pesquisadora no H\&TRC - Centro de Investigação em Saúde e Tecnologia; Professora do Departamento das Ciências Sociais e Humanas da Escola Superior de Tecnologia da Saúde de Lisboa do Instituto Politécnico de Lisboa, Lisboa, Portugal. teresa.denis@estesl.ipl.pt / http://orcid.org/0000-0002-4510-6779
}

http://dx.doi.org/10.1590/18094449201900550017

(c) $\mathrm{EY}-\mathrm{NC}$ 
Multiculturalism, Interculturalism, Human Rights and Gender

Violence: Brief Reflections on Female Genital Mutilation in Portugal and How to Address It

\begin{abstract}
Multiculturalism is an ever-growing reality in societies with increasing numbers of immigrants, where cities emerge as places of passage, encounter or cultural miscegenation, but also of social contrast or antagonism. Interculturalism appears as a tool for encouraging links with the idea of otherness, promoting integration and upholding the right to difference, while avoiding - or even denouncing - structures of domination over minorities. But this cultural diversity can only be protected, promoted and accepted as long as it does not question human rights or provoke exclusion or inequality. It is in this difficult realm, which is open to constant debate (and that frequently promotes polarizations), that we present brief notes that contribute to considering female genital mutilation in its inextricable condition as violence against women, based on the current Portuguese reality.
\end{abstract}

Keywords: Female Genital Mutilation, Interculturalism, Gender Violence, Human Rights, Portugal. 


\section{O Outro na cidade: da multiculturalidade à interculturalidade}

A tendência para a manifestação da multiculturalidade é hoje uma realidade crescentemente vivida nas sociedades de acolhimento de imigrantes, fazendo emergir cidades multiculturais enquanto lugares de passagem, encontro ou mestiçagem cultural, de rivalidade ou contraste social e mesmo de antagonismo $e$ crispação de cariz interétnico, inter-religioso ou socioeconómico, sobretudo quando estas circunstâncias se sobrepõem com ou suscitam situações de exclusão social, cultural e económica. Esta plataforma de potencial cruzamento e confronto multicultural, porém, por si só não implica a existência de contactos e interações significativas entre as culturas copresentes, que podem coexistir sem que haja lugar a trocas relevantes entre culturas, isto é, sem que se verifique efectivamente uma circunstância intercultural.

A interculturalidade coloca-se conceptualmente a um nível relacional e horizontal, remetendo idealtipicamente para a existência social de diferentes culturas e grupos que interagem entre si de diversos modos num quadro institucional e legal onde a cada um é conferido o direito à sua diferença, às suas práticas $e$ aos seus princípios morais, normativos e de conduta, que carecem de reconhecimento e respeito mútuos. O carácter essencialmente horizontal das relações interculturais fabrica-se, então, em termos idealtípicos, a partir de uma premissa social predominantemente não estratificante. ${ }^{1}$ Deste ponto de vista, uma abordagem intercultural é uma forma de ultrapassar a mera coexistência entre grupos, frequentemente assente em estruturas institucionais $e$ práticas de disputa ou exclusão, elaborando modos activos de procura de diálogo e de conhecimento do outro, e buscando uma intervenção política, cultural e social que substitua uma lógica de conflito por uma lógica de criatividade (Hernández, 2009). O objectivo almejado será sempre um esforço de integração que

1 Sobre o que liga e o que afasta os conceitos de multiculturalidade e interculturalidade, vejam-se, por exemplo, os contributos de Charles Taylor (2012), Gunther Dietz (2012), Nasar Meer e Tariq Modood (2012) ou de Martyn Barrett (2013). 
incorpore as diferenças de práticas, racionalidades e processos de subjectivação, alicerçando-se numa matriz intersubjectiva que reconheça a complexidade do que está em jogo e o jogue criticamente com as possibilidades e limites de variáveis como diálogo, divergência, antagonismo (Arpini, 2007).

$\mathrm{O}$ reconhecimento da multiculturalidade e o desígnio de uma integração intercultural têm vindo a fazer o seu caminho, apesar dos obstáculos e retrocessos, tanto no universo académico como, de modo não surpreendentemente mais tímido, no domínio das políticas públicas. E este caminho tem-se devotado essencialmente a propostas de pensar e agir sobre processos de exclusão e hegemonização sobre comunidades imigrantes ou indígenas sujeitas a processos históricos de submissão, aniquilamento ou aculturação. No quadro das possibilidades crescentemente oferecidas pela maior abertura e legitimação abstracta que o plano político das vontades e o plano legal das estruturas legais $e$ institucionais oferecem à expressão $e$ à salvaguarda da diversidade social e cultural, emerge um contexto que favorece maneiras concretas para que as práticas, as convicções e os modos de vida de cada comunidade se reforcem como factos sociais totais que evidenciam a complexidade própria dos modos de ser, estar, pensar e agir que definem determinado grupo, comunidade ou etnia.

Este cenário de maior reflexão e protecção, frequentemente apenas formal, pode contribuir para uma afirmação da coesão $e$ existência formal, material e imaterial dessas comunidades em moldes diversos daqueles que funcionavam para essas comunidades e para o contexto exterior, interferindo, perturbando $e$ actuando na configuração - embora de modo não inevitavelmente linear nem puro - das suas fronteiras identitárias. Nesta medida, a consolidação de políticas e práticas interculturais respaldadas na defesa dos direitos e da identidade do outro suscita um debate em torno do lugar que podem ocupar certas crenças $e$ rituais, associados por determinados grupos a eixos estruturantes de pertença cultural, gravitando o debate $-e$ a sua recorrente politização - em torno do confronto entre visões de protecção ou 
de denúncia do estatuto patrimonial dessas crenças e rituais no quadro de sociedades laicas e com uma moldura jurídica específica. O pomo joga-se frequentemente em torno da complexa discussão acerca da possibilidade ou impossibilidade de certos conjuntos de práticas culturais atentarem precisamente contra as bases de direitos nos quais se funda a matriz jurídica e institucional que fomenta a abordagem intercultural, mas também sobre os modos como a defesa de direitos culturais concretos pode comprometer objectivamente outros direitos (Stopler, 2005; Siobhán, 2006; Bhabha, 2009). Indiscutivelmente, a promoção de condições para a integração numa sociedade de características multiculturais ou, pelo menos, nas quais se verifique a existência pluricultural de grupos maioritários e minoritários, tem de assentar em pressupostos como a tolerância (no sentido de capacidade de interconhecimento e de reconhecimento da alteridade). As incidências concretas $e$ as consequências dessa tolerância na sua aplicabilidade apresentam, contudo, um conjunto complexo de limitações (Lopes, 2012). Assim, é neste campo, difícil e persistentemente aberto ao debate que nos propomos aduzir algumas notas acerca do caso da mutilação genital feminina (MGF) em Portugal, não raro apresentada por quem a defende e pratica como um facto de cultura.

\section{Mutilação genital feminina, violência de género $e$ direitos humanos: algumas notas sobre o caso português}

A possibilidade de mudança real de hábitos e mentalidades tende a ser marcada por lentidão e gradualidade, gerando focos de resistência amiúde capazes de persistir, sobretudo quando entra em confronto com crenças e relações de poder de base claramente assimétrica, como é o caso das diferenças de género produtoras de submissão e até violentação feminina (Pais, 1995; Pickup;Williams;Sweetman, 2001). Efectivamente a situação das mulheres imigrantes ou de minorias étnicoculturais nas sociedades desenvolvidas é determinada pela articulação de diferentes relações de poder em que se encontram inseridas. Neste âmbito, 
as abordagens feministas das migrações, por exemplo, têm salientado que a situação das mulheres migrantes passa por uma análise multidimensional que convoque as questões de género, classe social e pertença étnica, cultural, simbólica e profissional (Altamirano, 1997; Hondagneu-Sotelo, 2000; Silvey, 2004; Nawyn, 2010). Um dos aspectos concretos nos quais se cruzam e se materializam a desigualdade e a subalternização feminina é o da violência doméstica. Sublinhe-se que o fenómeno da violência doméstica possui um âmbito conceptual e analítico insusceptível de incorporar a MGF, que corresponde a um fenómeno inconfundível com aquele. A violência doméstica é classificada pela Organização Nações Unidas como

toda e qualquer ação de violência baseada no género que resulte, ou possa resultar, em danos ou sofrimento físicos, sexuais ou psíquicos das mulheres, inclusive ameaças, coerção ou privação sumária de liberdade, quer ocorra na vida pública ou privada (UN, 1993:artigo $1^{\circ}$ ).

No extremo desta problemática temos o caso da MGF, que a Organização Mundial de Saúde (OMS) definiu como qualquer procedimento que envolva a remoção total ou parcial dos órgãos genitais externos praticados por razões culturais, religiosas e nãoterapêuticas (OMS, 2009). Em termos especificamente procedimentais, a MGF consiste na remoção de parte ou de todos os órgãos sexuais externos femininos, praticada em mulheres adultas e em crianças entre os 4 e os 14/15 anos como acto de índole ritual ${ }^{2}$ por uma circuncisadora tradicional (fanateca) de entre os membros mais velhos da comunidade com a utilização de uma lâmina de corte, faca ou vidro cortante para remoção do

2 Não é demais salientar que historicamente, nem a excisão do clítoris se fez exclusivamente em países com comunidades muçulmanas, nem se fez por razões apenas religiosas. Veja-se, por exemplo, a prática de clitoridectomia e circuncisão feminina nos Estados Unidos da América como resposta clínica a problemas ou hábitos sexuais, como o onanismo ou a frigidez (Rodriguez, 2014). No século XIX há também referências a esta prática em Inglaterra e em França. 
clítoris ou até mesmo dos pequenos lábios com fundas consequências físicas e psicológicas traumáticas e também de saúde, sobrevindo infecções recorrentes, dor crónica, hemorragias dolorosas e complicações durante o parto, não raro com severidade (Osifo;Evbuomwan, 2009). ${ }^{3}$

Esta prática de natureza ritualística assenta num costume sociocultural de natureza patriarcal (Shweder, 2000), ${ }^{4}$ contando como procedimento clássico dos sistemas de dominação com a participação activa dos dominados (mulheres, que não raro ocupam até um lugar central na estrutura social de relações nos contextos de origem). O jaez consuetudinário desta prática estribase, por isso, nas desigualdades de género como mecanismo de controlo da sexualidade da mulher. ${ }^{5}$ É suportado por valores sobre pureza, modéstia e ascese que a mulher vista como impoluta deve preservar, pelo que é geralmente iniciada e executada por mulheres que a vêem como motivo e desejo de inclusão social, ou receio de rejeição pela comunidade. Manuela Cunha salienta a

3 Segundo a OMS (2009:6), existem quatro tipos de MGF, designadamente o tipo I, remoção total ou parcial do clítoris (clitoridectomia); o tipo II, remoção total ou parcial do clítoris e dos pequenos lábios (excisão), com ou sem excisão dos grandes lábios; o tipo III, oclusão ou estreitamento do canal vaginal, cortando e apondo os grandes ou os pequenos lábios, com ou sem excisão do clítoris (infibulação); e o tipo IV, todas as restantes intervenções sobre a genitália feminina por razões extra-clínicas, envolvendo perfuração, colocação de piercing, incisão, punção, escarificação e cauterização.

${ }^{4}$ Não obstante constituir uma categoria assente num sistema classificatório $e$ taxonómico objectivável metodologicamente, o termo "patriarcal" pode resultar igualmente ou ser visto como resultando de uma visão de proveniência ocidental de timbre essencialista, exoticizada e paternalista sobre a mulher não ocidental, representada a priori como vítima de opressão e de ignorância (Cunha, 2013). É, pelo menos, esta a denúncia de um conjunto de feministas africanas relativamente ao um activismo feminista euroamericano, acusado de aspiração salvífica e de deslegitimar as narrativas de mulheres excisadas que não radique na assunção de vitimização (Parmar; Walker, 1996).

${ }^{5}$ Mesmo que seja possível encontrar na ancestralidade desta prática outro tipo de razões que expliquem a sua existência, como a protecção contra o estupro (Gruenbaum, 2000), a MGF constitui nas circunstâncias actuais um óbvio instrumento de subalternização feminina violenta. 
necessidade de problematizar a MGF como conjunto de práticas situadas em universos de significação muito distintos. Recorda a autora que

sob a terminologia MGF esconde-se uma diversidade de realidades, sentidos e experiências. Consoante o contexto em que decorram $e$ as relações de poder envolvidas, para algumas mulheres é algo traumático e violento, com sequelas sérias na sua saúde sexual e reprodutiva; outras encaram-na como uma experiência positiva $e$ até empoderadora [...], mesmo que, à semelhança de tantos outros rituais de iniciação pelo mundo fora - femininos $e$ masculinos -, fisicamente dolorosa (Cunha, 2013:843). ${ }^{6}$

Apesar da multiplicidade de formas por via das quais se exerce a MGF e da variabilidade de contextos de simbolização e valorização em que essa prática ocorre, o que lhe confere uma particular complexidade e pluralidade fenomenológicas e interpretativas, o facto é que a crença na bondade do acto ablativo $e$ a representação da sua inevitabilidade conferem-lhe uma capacidade socialmente reprodutiva muito vincada, estando a prática de mutilação "no centro de toda a dinâmica social" dos grupos que a ela recorrem (Erlich, 1986:271).

Apresentando-se como um facto de natureza cultural, a MGF apela abstractamente, dir-se-ia, a hermenêticas particularistas assentes nas diferenças socioculturais inerentes ao relativismo cultural, cuja caricatura interditaria a escolha ou o julgamento desvalorizador, tidos na caricatura como deslegitimadores $e$ etnocêntricos. Fora da redução adulteradora da posição relativista, é evidente que no plano ético qualquer atentado contra a vida humana ou a integridade física se coloca num patamar superior à

${ }^{6}$ Prossegue conclusivamente a autora: "Impõe-se por isso a cautela metodológica de atender a esta diversidade de realidades e não decidir à partida que as mulheres que não se consideram vitimizadas por estas práticas não podem senão estar confusas, ou ser vítimas de falsa consciência" (Cunha, 2013:843). 
aceitação acrítica - e, portanto, contra-relativista - do factor cultural ou étnico definido por si próprio. Nesse sentido, as diferenças culturais só podem ser aceites no limite ético da dignidade humana, incompatível com a violação da vida e da integridade física e mental dos seres humanos, sejam mulheres ou homens. ${ }^{7} \mathrm{O}$ choque entre a preponderância do direito à diferença $e$ a preponderância do direito à igualdade ilustra-se na problemática da MGF, fazendo sobrevir nas sociedades com níveis mais elevados de desenvolvimento económico, social e político, de diversidade cultural crescente, uma negociação e uma definição permanentes dos seus limites (Santos, 2002) à luz dos adquiridos éticos institucionalizados e formalizados nos direitos humanos. Reconhecendo um intervalo de debate cujas fronteiras são amplas $e$ movediças, remetendo para aspectos de articulação que constituem uma espécie de negociação entre práticas culturais auto $e$ hetero-definidas enquanto tal e direitos reivindicados $e$ disputados (Hernlund; Shell-Duncan, 2007), assente-se no consenso de que as questões étnico-culturais não podem sobrepor-se aos direitos humanos. Entendendo que os direitos humanos são construções sociais, eles podem ser objecto de relativização social e cultural, mas nos limites reconhecidos pela ética, ela própria igualmente engendrada socialmente.

Assim, a diversidade cultural só pode ser protegida, promovida $e$ aceite desde que não coloque em causa os direitos humanos, quer a nível das liberdades fundamentais, como por exemplo, a liberdade de expressão, quer a nível da integridade física e mental ou do direito à vida. Ou seja, como estipula a Convenção Sobre a Protecção e a Promoção da Diversidade das Expressões Culturais emanada pela Conferência Geral da Organização das Nações Unidas para a Educação, a Ciência e a Cultura (UNESCO) em 2005, ninguém poderá invocar a

\footnotetext{
7 As limitações e ambivalências desta asserção devem, no entanto, ser reconhecidas e assumidas, sendo mais visíveis nas múltiplas interrogações levantadas pela retórica ou justificação de saúde ao próprio conceito de ética e à operacionalidade das suas fronteiras (Palhares; Squinca, 2013).
} 
diversidade cultural para atentar contra os direitos e liberdades garantidos pelo direito internacional (UNESCO, 2005:artigo $2^{\circ}$ ).

Em todo o caso, o ritual existe, ${ }^{8}$ afectando actualmente muitos milhões de mulheres (ascendendo, segundo cálculos da Organização Mundial de Saúde, a 200 milhões de mulheres - WHO, 2018), ${ }^{9}$ e é praticado de modo habitual e corrente em cerca de uma trintena de países (WHO, 2014; 2018; Teixeira;Lisboa, 2016), localizados sobretudo na região subsaariana do continente africano (mas atingindo latitudes tão diversas quanto a Colômbia, a Malásia ou Israel), entre os quais Guiné-Bissau, país lusófono que integra este lote, e no seio do qual se verifica uma prática ritualística de MGF, essencialmente concentrada em algumas etnias predominantemente islâmicas e que constituem uma parte bastante significativa da população que corporiza esta prática tanto na Guiné-Bissau como nas comunidades radicadas em Portugal (Johnson, 2007; Oberreiter, 2008; Piedade, 2008; Martingo, 2009; Lisboa et alii, 2016). Sublinhe-se, todavia, que no que concerne à MGF, procurar nexos causais automáticos entre a pertença religiosa $e$ a adopção dos rituais de mutilação é um exercício arriscado do ponto de vista de uma hermenêutica científica do fenómeno, podendo conduzir a reificações $e$ determinismos cuja base não encontra documentação de suporte e que carreariam como consequência até não intencional o reforço de posicionamentos de base xenófoba no seio de um debate já de si "tão polarizado e carregado de imputações ideológico-morais" (Cunha, 2013:836). Com efeito, e circunscrevendo a análise apenas à África, o polimorfismo social e cultural da MGF e das suas motivações transforma a sua "prática num comportamento

\footnotetext{
8 Não obstante vislumbrar-se um declínio na última década e meia, principalmente em determinadas regióes onde se verificaram alguns pressupostos de actuação pública e implicação das comunidades, como os Yoruba do sudoeste nigeriano (Caldwell; Orubuloye; Caldwell, 2000).

9 Outras fontes sugerem uma cifra diferente de afectadas. Ainda assim, para P. Stanley Yoder, Shanxiao Wang e Elise Johansen (2013), por exemplo, no início desta década o número de mulheres vivas submetidas a MGF rondaria os 87 milhões.
} 
prosseguido por praticantes de todas as grandes crenças religiosas do continente - cristianismo, islamismo e as religióes tradicionais" (Odukogbe et alii, 2017:139). Carla Martingo, por exemplo, que estudou a MGF na população guineense, não deixa de sublinhar que "o corte dos genitais femininos observa-se tanto em populações muçulmanas, como em judeus e cristãos, mas com particular incidência em populações islamizadas" (Martingo, 2009:165); até porque se trata de uma prática bem anterior ao surgimento do Islão.

Nos bairros ou comunidades onde residem guineenses em Portugal constata-se que estas etnias se tendem a fechar sobre si próprias, gerando uma estrutura comunitária e relacional pouco porosa a redes exteriores de sociabilidade. Facilitando uma eventual perpetuação desta prática, os casamentos são predominantemente endogâmicos e intra-étnicos como forma de manter a coesão familiar, cultural e simbólica ligada aos valores de origem, o que reforça o carácter de silenciamento sobre a prática da MGF e sobre quem a vive e a ela é submetida. Convém lembrar, todavia, que continua a ser difícil afirmar se há uma prática de MGF perceptível e reiterada em Portugal (Martingo, 2009; Lisboa et alii, 2016), ainda que em 2016 se tenha estimado - a partir de dados dos Censos de 2011 - em 1830 o número de mulheres/meninas com 15 anos ou menos sujeitas de facto ou potencialmente sujeitas a MGF (Teixeira; Lisboa, 2016).

Manifestar-se contra este costume pode ser, por isso, difícil tanto para as mulheres como para os homens que, do interior do grupo, questionam a legitimidade ou mesmo que se manifestam contra tal prática. Os posicionamentos críticos e de denúncia da parte de membros das próprias comunidades onde se pratica a MGF podem tender a ser assimilados a efeitos de dissolução da sua integridade sociocultural e da imobilidade que a sustenta. Um dos maiores obstáculos à mudança erigida a partir da acção de membros do grupo reside, pois, nesta dimensão de autorepresentação contracultural. Adoptando uma terminologia habermasiana, é como se a acção organizada de mulheres que sofreram MGF ou de outros membros da comunidade que a 
pratica fosse perspectivada como fazendo parte do sistema que se oporia ao mundo de vivência a que pertenceriam o quotidiano e a identidade de comunidade em si (Habermas, 1984, 1987, 1990).

Recorrendo, uma vez mais, ao exemplo de membros da comunidade guineense radicada em Portugal (mas também de outras comunidades nacionais imigrantes), e no âmbito da auscultação de mulheres que foram sujeitas enquanto crianças à MGF, estudos como o de Sandra Piedade (2008) ou o de Edna Silva (2012) desenham um cenário entranhado no silêncio, na vergonha, na conformação e no trauma, expressando muitas das mulheres entrevistadas ou inquiridas elementos axiais à sua identidade respaldados no medo, na inibição $e$ na humilhação $e$ vivendo quotidianos de supressão de sentimentos e de uma autoimagem feita de um percurso identitário deteriorado ou interrompido, no qual a MGF constitui um marco central no seu desenvolvimento psicossocial. Ambos os estudos dão igualmente conta de uma transformação em curso, sobretudo em mulheres jovens submetidas à MGF, no modo como muitas se dizem dispostas a interromper o ciclo de reprodução social de uma prática que aprenderam a criticar. A mudança decorrerá essencialmente do aumento da escolaridade e da sua consequência socializadora (que não deve ser menosprezada), resultando também de uma exposição de efeito eventualmente socializador (embora ocorrendo frequentemente numa matriz de confronto com valores e crenças anteriores e possuindo, além disso, eficácia variável e difícil de aferir) aos valores sociais dominantes da sociedade de acolhimento e das estruturas de intervenção dos poderes públicos.

\section{Da resposta necessária aos limites, ambivalências e desafios}

Mas a prática é consuetudinária e revela poder de continuidade, ilustrando como caso a participação da dominada na manutenção social do regime que a oprime. No estudo que conduziu em torno dos discursos e das representações produzidas por elementos da comunidade guineense em Portugal, por 
exemplo, Edna Silva (2012) afirma existir um número significativo de mães, tias ou avós que assumem fazer o que pensam ser o melhor para as suas filhas, sobrinhas ou netas de acordo com a tradição, que concebem como benéfica e necessária para as possibilidades matrimoniais das próprias mutiladas. Evidentemente que a complexidade da realidade da MGF não se altera automaticamente porque se criou um aparato legislativo ou porque se inscreveu o problema na agenda institucional de estruturas decorrentes de decisões políticas, ${ }^{10}$ passos, todavia, imprescindíveis para que se possam obter resultados. Concretizar o desiderato político faz-se proveitosamente - embora com graus diversos de eficácia e num quadro que tem inevitavelmente de se estender no tempo - incluindo pressupostos de instauração $e$ promoção do diálogo intercultural, abrindo com essa experiência espaços de reflexão e análise da interacção interventiva com as populações envolvidas. Reconhecendo que esta formulação reúna laivos de truísmo, frequentemente não se estabelecem condições práticas, logísticas e humanas, suportadas institucional $e$ formacionalmente, para a configuração de espaços de relação que, mais do que punir ou impor, ${ }^{11}$ possibilitem efectivamente uma capacitação e um empoderamento das mulheres ${ }^{12}$ para que estas passem a não deixar que as suas filhas sejam mutiladas.

\footnotetext{
${ }^{10}$ De que serão exemplo os Programas de Acção para a Prevenção e Eliminação da Mutilação Genital Feminina. Vejam-se os três programas aprovados pelo governo português e mais uma dezena e meia de documentos relativos à MGF [https:/www.cig.gov.pt/documentacao-de-referencia/legislacao/mutilacao-genitalfeminina/ e em https:/www.cig.gov.pt/documentacao-dereferencia/doc/mutilacao-genital-feminina/ - acesso em 30 mar 2019].

${ }^{11}$ Mesmo assumindo que o poder das políticas públicas se traduzisse numa real capacidade de capilarização e penetração nos meios sociais onde este tipo de prática é - ou pode potencialmente ser - levado a cabo em Portugal, haveria sempre a eventualidade, senão probabilidade, de que as mulheres/meninas fossem submetidas a MGF, por exemplo, no decurso de uma viagem de férias à Guiné-Bissau.

${ }^{12}$ As condições que propiciem o empoderamento, juntamente com aquelas que fomentem os níveis de escolarização das mulheres, são factores de rejeição da MGF (Rahlenbeck;Mekonnen, 2009).
} 
O pano de fundo da intervenção deverá evitar a trivialização da cultura, consistindo esta posição na ideia de que as pessoas estão presas irremediavelmente a princípios socioculturais avassaladores $e$ inescapáveis, tornando-as incapazes de se conceber fora dos eixos de significação cultural que recortam o corpo de uma certa forma, tornando-o aceitável ou mesmo desejável. Esta asserção, já de si discutível nos contextos de origem, torna-se muito mais difícil de defender em contextos multiculturais de sociedades de acolhimento capazes de suscitar múltiplas pertenças e de fornecer um cenário caleidoscópico de fluidez entre comunidades que à partida se poderiam supor como fechadas. Mas a intervenção deverá igualmente reconhecer a complexidade e a variabilidade de práticas, que frequentemente correspondem à sua adopção por mulheres adultas, associandolhes mundivisões de relação intrínseca entre um corpo saudável $e$ sexualizado como resultado de procedimentos nem sempre facilmente inseríveis na ampla categoria de MGF (Bagnol; Mariano, 2008a), como as práticas vaginais de mulheres de certas zonas e grupos em Moçambique, por exemplo (Bagnol; Mariano, 2008b).

É nesse sentido que Manuela Cunha advoga a destrinça entre os direitos das crianças $e$ adolescentes e os direitos das mulheres adultas, que uma criminalização cega e universal da MGF não prevê, já que nega a mulheres legalmente emancipadas uma opção de prosseguimento de alterações corporais segundo os seus próprios termos (Cunha, 2013). A necessidade de protecção dos direitos das crianças e adolescentes deve, por isso, ser pensada à luz de uma rejeição da alterização do constrangimento social, segundo a qual só há MGF quando se trata de membros de certa origem étnica, nacional ou religiosa, retirando desta conotação negativa e estigmatizada todo o conjunto de práticas de intervenção medicalizada nos genitais, vista como legítima $e$ aceitável e, portanto, normalizada. Com efeito,

o discurso dominante sobre as cirurgias de cosmética genital associa-as não à MGF mas a outras cirurgias cosméticas socialmente aceites (e. g. nariz, peito) e remete- 
as para o quadro do direito das mulheres à livre escolha e a tomar decisões sobre o seu corpo (...) [o que pode redundar no] risco de discriminarmos entre genitais "europeus" e "africanos" (Cunha, 2013:850).

O desafio é de natureza simultaneamente radicada, por um lado, na necessidade de produção e utilização de conhecimento sociológico, antropológico e histórico, e, por outro, na indispensabilidade de aplicação de um plano de formação de profissionais habilitados especificamente para a detecção, reconhecimento e intervenção em famílias e crianças em risco. Conhecer a realidade do fenómeno em Portugal, enquadrar as razões socioculturais e os sentidos simbólicos das práticas, contemplar o jaez intrincado e multímodo da interferência do género e da classe social ${ }^{13}$ na forte hierarquização da condição da mulher imigrante ou das minorias, perceber a posição dos pais sobre a MGF, sem julgar, transmitir respeito pelas suas práticas tradicionais, desaprovar com subtileza e empatia a mutilação explicando as consequências físicas e psíquicas para as crianças, educar sobre a anatomia dos órgãos genitais femininos, explicar as complicações do ato no presente e no futuro ou reconhecer a representatividade de dirigentes locais e mobilizar a sua vontade de participação são competências fulcrais num processo de formação de profissionais para que se possa ambicionar a geração de um processo de mudança social e o encorajamento do abandono desta prática, que além de materialmente constituir uma violação dos direitos humanos, coloca crianças e mulheres em perigo de vida, provocando sentimentos de baixa autoestima, depressão, ansiedade, fobias, pânico e desordens psicóticas de variada ordem.

Ou seja, a análise e a acção dela decorrente devem suscitar o reconhecimento de que a tendência para uma realidade multicultural é uma constatação que em si mesma engendra

${ }^{13}$ A ruralidade e a condição socioeconómica pouco elevada são variáveis favoráveis à reprodução social da prática ablativa (Silva, 2012). 
limites, ambivalências e desafios que não podem ser ignorados, dado o seu potencial de tensão nas relações de geometria variável entre comunidades, na relação entre indivíduo reconhecido com entidade cívica e formal e comunidade reconhecida na sua identidade colectiva e no seu património (Touraine, 1992), e nos modos como esses múltiplos patamares de relações podem ser contextualizados normativamente, tanto no nível do sistema jurídico-legal em vigor como no nível da realidade portuguesa como estrutura social complexa. Neste sentido, é preciso alertar e formar professores, sociólogos, antropólogos, assistentes sociais, médicos, enfermeiros, técnicos de saúde e mediadores sociais para que se passe definitivamente do reconhecimento meramente celebrativo do multiculturalismo como produto da modernidade, não muito distante de propostas como a de Brian Barry (2001), num movimento que, de resto, não é de agora no plano das propostas de campos do conhecimento como a sociologia, a antropologia ou a história, para a consecução de um diálogo intercultural que obrigue à troca entre hábitos e mundividências, frequentemente antitéticas, diálogo esse inelutavelmente atravessado pela difícil relação entre posições hegemónicas $e$ contra-hegemónicas. Um desafio gigante e certamente mais fácil de inserir num registo de conceptualização do que de concretização.

\section{Referências bibliográficas}

AltamiRANO, Aha Tollefsen. Feminist theories and migration researchmaking sense in the data feast? Refuge: Canada's Journal on Refugees/Revue Canadienne sur les Réfugiés, vol. 16, ${ }^{\circ} 4$, Toronto, Centre for Refugee Studies of York University, 1997, pp.4-8.

ARPINI, Adriana. Diversidad y conflictividad. Contribuciones a la formulación de categorías heurísticas para la integración y el diálogo intercultural. In: HERCEG, José Santos (org.). Integración y Interculturalidad: desafíos pendientes para América Latina. Santiago do Chile, Instituto de Estudios Avanzados da Universidad Santiago de Chile, 2007, pp.15-30. 
BAGNOL, Brigitte; MARIANO, Esmeralda. Elongation of the labia minora and use of vaginal products to enhance eroticism: can these practices be considered FGM? The Finnish Journal of Ethnicity and Migration, vol. 3, no 2, Helsínquia, Society for the Study of Ethnic Relations and International Migration (ETMU), 2008a, pp.42-53.

BAGNOL, Brigitte; MARIANO, Esmeralda. Vaginal practices: eroticism and implications for women's health and condom use in Mozambique. Culture, Health \& Sexuality, vol. 10, $\mathrm{n}^{\circ}$ 6, Lima, International Association for the Study of Sexuality, Culture \& Society, 2008b, pp.573-585.

BARRETT, Martyn (org.). Interculturalism and multiculturalism: similarities and differences. Estrasburgo, Council of Europe Publishing, 2013.

BARRY, Brian. Culture and equality: an egalitarian critique of multiculturalism. Cambridge, MA, Harvard University Press, 2001.

BHABHA, Faisal. Between exclusion and assimilation: experimentalizing multiculturalism. McGill Law Journal, vol. 54, $\mathrm{n}^{\circ}$ 1, Montreal, [Student body of] Faculty of Law of McGill University, 2009, pp.4590.

Caldwell, John C.; Orubuloye, I. O. e Caldwell, Pat. Female genital mutilation: conditions of decline. Population Research and Policy Review, e Nova Iorque, Southern Demographic Association e Springer, vol. 19, n 3, 2000, pp.233-254.

CUNHA, Manuela Ivone. Género, cultura e justiça: a propósito dos cortes genitais femininos. Análise Social, vol. 48, n 209, Lisboa, Instituto de Ciências Sociais, 2013, pp.834-856.

DIETZ, Gunther. Multiculturalismo, interculturalidad y diversidad en educación. Una aproximación antropológica. Cidade do México, Fondo de Cultura Económica, 2012.

ERLICH, Michel. La femme blessée. Essai sur les mutilations sexuelles féminines. Paris, L'Harmattan, 1986.

GRUENBAUM, Ellen. Is female "circumcision" a maladaptive cultural pattern? In: SHELL-DuNCAN, Bettina; HERnLUnd, Ylva (org.). Female "circumcision" in Africa. Culture, controversy and change. Londres, Lynne Rienner Publishers, 2000, pp.41-54. 
HABERMAS, Jürgen. The theory of communicative action. Vol. 1, Reason and the rationalization of society. Boston, Beacon Press, 1984.

HABERMAS, Jürgen. The theory of communicative action. Vol. 2, Lifeworld and system: a critique of functionalist reason. Boston, Beacon Press, 1987.

HABERMAS, Jürgen. Moral consciousness and communicative action. Cambridge, MA, Massachusetts Institute of Technology Press, 1990.

HERNÁNDEZ, Natalio. De la exclusión al diálogo intercultural con los pueblos indígenas. Cidade do México, Plaza y Valdés Editores e Universidad Intercultural de Chiapas, 2009.

HeRnlund, Ylva; SHELl-DunCAN, Bettina (org.). Transcultural bodies. Female genital cutting in global context. New Brunswick, NJ, Rutgers University Press, 2007.

Hondagneu-Sotelo, Pierrette. Feminism and migration. The Annals of the American Academy of Political and Social Science, vol. 571, Filadélfia, American Academy of Political and Social Science, 2000, pp.107-120.

JOHNSON, Michelle C. Making Mandinga or making Muslims? Debating female circumcision, ethnicity, and Islam in Guinea-Bissau and Portugal. In: SHELl-DUNCAN, Bettina; HERNLUND, Ylva (org.). Transcultural bodies. Female genital cutting in global context. New Brunswick, NJ, Rutgers University Press, 2007, pp.202-223.

LISBOA, Manuel et alii. Mutilação genital feminina em Portugal. Prevalências, dinâmicas socioculturais e recomendaçóes para a sua eliminação. Vila Nova de Famalicão, Húmus, 2016.

LOPES, Ana d'Ávila. Da coexistência à convivência com o outro: entre o multiculturalismo e a interculturalidade. REMHU: Revista Interdisciplinar da Mobilidade Humana, vol. 20, $\mathrm{n}^{\circ} 38$, Brasília, Centro Scalabriniano de Estudos Migratórios, 2012, pp.67-81.

MARTINGO, Carla. O corte dos genitais femininos em Portugal: o caso das guineenses - estudo exploratório. Lisboa, Alto-Comissariado para a Imigração e Diálogo Intercultural, 2009.

MEER, Nasar; MoDOOD, Tariq. How does interculturalism contrast with multiculturalism? Journal of Intercultural Studies, vol. 33, $\mathrm{n}^{\circ} 2$, 
Burwood, Vic, Alfred Deakin Institute for Citizenship and Globalisation da Deakin University, 2012, pp.175-196.

MullaLly, Siobhán. Gender, culture and human rights: reclaiming universalism. Oxford e Portland, Hart Publishing, 2006.

NAWYN, Stephanie J. Gender and migration: integrating feminist theory into migration studies. Sociology Compass, vol. 4, $\mathrm{n}^{\circ}$ 9, Hoboken, NJ, John Wiley \& Sons Ltd., 2010, pp.749-765.

OBERREITER, Julia Anna. A cut for a lifetime. The case of female genital mutilation among the community of Guinea Bissau in Lisbon. Dissertação (Mestrado em Direitos Humanos e Democratização), Universidade Nova de Lisboa, Lisboa, 2008.

ODUKOGBE, Akin-Tunde A. et alii. Female genital mutilation/cutting in Africa. Translational Andrology and Urology, vol. 6, n 2, Hong Kong, AME Publishing Company, 2017, pp.138-148.

ORGANIZAÇÃO das Nações Unidas para a Educação, a Ciência e a Cultura (UNESCO). Convenção sobre a protecção e a promoção da diversidade das expressóes culturais. Adoptada na 33. ${ }^{\text {a Sessão da }}$ Conferência Geral da UNESCO, em Paris, a 20 de Outubro de 2005 [https://www.unescoportugal.mne.pt/images/Comunica\%C3\%A7\%C3 \%A3o/convencao_sobre_a_proteccao_e_a_promocao_da_diversidad e_das_expressoes_culturais.pdf - acesso em 30 mar 2019].

ORGANIZAÇÃO Mundial de Saúde (OMS). Eliminação da mutilação genital feminina: declaração conjunta OHCHR, ONUSIDA, PNUD, UNECA, UNESCO, UNFPA, ACNUR, UNICEF, UNIFEM, OMS. S.L., Associação para o Planeamento da Família, 2009.

OSIFO, David Osarumwese; EvBUOMWAN, Iyekoretin. Female genital mutilation among Edo people: the complications and pattern of presentation at a pediatric surgery unit, Benin City. African Journal of Reproductive Health/La Revue Africaine de la Santé Reproductive, vol. 13, $\mathrm{n}^{\circ} 1$, Cidade de Benin, Women's Health and Action Research Centre, 2009, pp.17-25.

PAIS, Marta Santos. Direitos humanos: violência contra as mulheres. In: Secretaria de Estado da Justiça. Os direitos humanos e a violência contra as mulheres. Lisboa, Secretaria de Estado da Justiça, Universidade Católica Portuguesa, Comissão para a Igualdade e para 
os Direitos das Mulheres e Clube Soroptimista Internacional de Lisboa, 1995, pp.33-41.

PALHARES, Dário; SQUINCA, Flávia. Os desafios éticos da mutilação genital feminina e da circuncisão masculina. Revista Bioética, vol. 21, $\mathrm{n}^{\circ} 3$, Brasília, Conselho Federal de Medicina, 2013, pp.432-437.

PARMAR, Pratibha; WALKER, Alice. Warrior masks. Female genital mutilation and the sexual blinding of women. Nova Iorque, Harcourt Brace \& Company, 1996.

PICKUP, Francine; Williams, Suzanne e SWEETMAN, Caroline. Ending violence against women. A challenge for development and humanitarian work. Oxford, Oxfam, 2001.

PIEDADE, Sandra. Mutilação genital feminina em Portugal. Dissertação (Mestrado em Psicologia Social e das Organizações), Instituto Superior de Ciências do Trabalho e da Empresa de Lisboa, Lisboa, 2008.

RAHLENBECK, Sibylle I.; MEKONNEN, Wubegzier. Growing rejection of female genital cutting among women of reproductive age in Amhara, Ethiopia. Culture, Health \& Sexuality, vol. 11, $\mathrm{n}^{\circ} 4$, Lima, International Association for the Study of Sexuality, Culture \& Society, 2009, pp.443-452.

RODRIGUEZ, Sarah B. Female circumcision and clitoridectomy in the United States: a history of a medical treatment. Suffolk e Rochester, NY, Boydell \& Brewer e University of Rochester Press, 2014.

SANTOS, Boaventura Sousa. Para uma sociologia das ausências e uma sociologia das emergências. Revista Crítica de Ciências Sociais, $\mathrm{n}^{\circ}$ 63, Coimbra, Centro de Estudos Sociais da Universidade de Coimbra, 2002, pp.237-280.

SHWEDER, Richard A. What about "Female genital mutilation"? And why understanding culture matters in the first place. Daedalus, Journal of the American Academy of Arts and Sciences, vol. 129, $\mathrm{n}^{\circ}$ 4, Cambridge, MA, American Academy of Arts and Sciences, 2000, pp.209-232.

SILVA, Edna. Discursos e representações sobre a prática da mutilação genital feminina na comunidade guineense em Portugal. Dissertação 
(Mestrado em Antropologia), Universidade Nova de Lisboa, Lisboa, 2012.

SILVEY, Rachel. Power, difference and mobility: Feminist advances in migration studies. Progress in Human Geography, vol. 28, $\mathrm{n}^{\circ} 4$, Newbury Park, CA, Sage Publications, 2004, pp.1-17.

STOPLER, Gila. The liberal bind: the conflict between women's rights and patriarchal religion in the liberal state. Social Theory and Practice, vol. 31, $\mathrm{n}^{\circ}$ 2, Tallahassee, Florida State University Department of Philosophy, 2005, pp.191-231.

TAYLOR, Charles. Interculturalism or multiculturalism? Philosophy \& Social Criticism, vol. 38, $\mathrm{n}^{\circ} 4-5$, Newbury Park, CA, Sage Publications, 2012, pp.413-423.

TEIXEIRA, Ana Lúcia; LISBOA, Manuel. Estimating the prevalence of female genital mutilation in Portugal. Public Health, vol. 139, Londres, The Royal Society for Public Health, 2016, pp.53-60.

TOURAINE, Alain. Critique de la modernité. Paris, Fayard, 1992.

UNITED Nations (UN). Declaration on the elimination of violence against women. Resolution 48/104 of 20 December 1993 [http://www.un.org/documents/ga/res/48/a48r104.htm - acesso em 30 mar 2019].

WORLD Health Organization (WHO). Female Genital Mutilation. 31 January $2018 \quad$ [https://www.who.int/en/news-room/factsheets/detail/female-genital-mutilation - acesso em 30 mar 2019].

WORLD Health Organization (WHO). Female genital mutilation (FGM). Prevalence of FCG. 2018 [http://www.who.int/reproductivehealth/topics/fgm/prevalence/en/ acesso em 30 mar 2019].

YODER, P. Stanley; WANG, Shanxiao e JOHANSEN, Elise. Estimates of female genital mutilation/cutting in 27 African countries and Yemen. Studies in Family Planning, vol. 44, $\mathrm{n}^{\circ}$ 2, Nova Iorque, Population Council, 2013, pp.189-204. 